\title{
ON THE STRUCTURE OF LINDENBAUM ALGEBRAS: AN APPROACH USING ALGEBRAIC LOGIC
}

\author{
CHARLES PINTER
}

\begin{abstract}
The following problem of algebraic logic is investigated: to determine those Boolean algebras which admit the structure of a nondiscrete cylindric algebra. A partial solution is found, and is then used to give an algebraic characterization of the Lindenbaum algebras of formulas of several broad classes of countable theories.
\end{abstract}

1. Introduction. A major open problem of algebraic logic is the following: Which Boolean algebras admit the structure of a nondiscrete cylindric or polyadic algebra? Using results of Henkin, Monk and Tarski [1], one easily proves:

A denumerable Boolean algebra admits the structure of a nondiscrete, dimension-complemented cylindric algebra if and only if it is not atomic.

We establish this, as well as a few related results, and use them to investigate the structure of Lindenbaum algebras of countable theories.

In the sequel, let $T$ denote any countable theory. By the Lindenbaum algebra $\mathscr{F}_{T}$ of $T$ we will always mean the Lindenbaum algebra of formulas ${ }^{1}$ of $T$. From results in [1] we easily establish that if $T$ has no one-element models then $\mathscr{F}_{T}$ is atomless (this characterizes $\mathscr{F}_{T}$, for there is, up to isomorphism, only one atomless, denumerable Boolean algebra). Let us say that $T$ admits elimination of all but $n$ predicates if $T$ is definitionally equivalent to a theory $T^{\prime}$ whose language may have finitely or denumerably many operation symbols, but has no more than $n$ predicate symbols other than $=$; for $n=0$, we say that $T$ admits elimination of predicates. It is shown that an arbitrary theory $T$ admits elimination of predicates if and only if $T$ has either no one-element models, or all of its one-element models are elementarily equivalent. We prove that if a theory $T$ admits elimination of predicates then $\mathscr{F}_{T}$ has $\leqslant 1$ atom; more generally, if $T$ admits elimination of all but $n$ predicates then $\mathscr{F}_{T}$ has $\leqslant 2^{n}$ atoms. Then we provide a method to determine the exact structure of $\mathscr{F}_{T}$ whenever $T$ admits elimination of all but finitely many predicates.

Our notation and terminology is that of Henkin, Monk and Tarski [1], and we presuppose an acquaintance at least with Chapter 1 of this work.

\footnotetext{
Received by the editors April 15, 1974 and, in revised form, April 16, 1975.

AMS (MOS) subject classifications (1970). Primary 02J15.

1 We will deal here with Lindenbaum algebras of formulas, rather than Lindenbaum algebras of sentences.
} 
2. Results on cylindric algebras. Throughout this section, let $\mathfrak{A}=\langle A,+, \cdot,-$, $\left.0,1, c_{\kappa}, d_{\kappa \lambda}\right\rangle_{\kappa, \lambda<\alpha}$ be a nondiscrete, dimension-complemented cylindric algebra. By $[1,1.11 .3(\mathrm{iii})], \alpha \geqslant \omega$. The following statements, which are easily deduced from results given in [1], will be needed in the sequel:

(A) if $c_{0}^{\partial} d_{01}=0$, then $\mathfrak{A}$ is atomless;

(B) if $c_{0}^{\partial} d_{01} \neq 0$, and there is no zero-dimensional element $x \neq 0$ such that $x<c_{0}^{\partial} d_{01}$, then $c_{0}^{\partial} d_{01}$ is the only atom of $\mathfrak{A}$;

(C) $\mathfrak{A} \cong \mathfrak{B} \times \mathbb{C}$ where $\mathfrak{B}$ is atomless and $\mathbb{C}$ is discrete.

(A) and (B) follow from [1, Theorems 1.10.5(ii), 1.11.8(i) and 1.6.20 ${ }^{2}$. (C) follows from [1, Theorems 2.4.37 and 1.11.8(ii)].

Every Boolean algebra admits the structure of a discrete cylindric algebra, so there is no need to consider that case further. Similarly, every Boolean algebra admits the structure of a cylindric algebra of degree 1 , for example by taking $c$ to be the quantifier given by $c 0=0, x \neq 0 \Rightarrow c x=1$. Thus, we should confine our attention to nondiscrete cylindric algebras of degree $\alpha \geqslant 2$.

If $\mathfrak{B}$ is any denumerable, atomless Boolean algebra, then $\mathfrak{B}$ admits the structure of a nondiscrete cylindric algebra of degree $\omega$. Indeed, if $T$ is any countable theory which has no one-element models, then $T \vdash \neg\left(\forall v_{0}\right)$ $\left(v_{0}=v_{1}\right)$, hence by (A), the Lindenbaum algebra of formulas of $T$ is an atomless denumerable Boolean algebra. This Lindenbaum algebra is isomorphic to $\mathfrak{B}$ because any two denumerable atomless Boolean algebras are isomorphic.

Now, let $\mathfrak{A}$ be any denumerable Boolean algebra having a direct factor which is an atomless denumerable Boolean algebra, say $\mathfrak{A} \cong \mathfrak{B} \times \mathfrak{C}$ where $\mathfrak{B}$ is atomless and denumerable. We have just seen that $\mathfrak{B}$ admits the structure of a nondiscrete, dimension-complemented cylindric algebra, and t5 certainly admits the structure of a discrete cylindric algebra, hence $\mathfrak{A}$ admits the structure of a nondiscrete, dimension-complemented cylindric algebra. Combining this with $(\mathrm{C})$, we get

(D) A denumerable Boolean algebra admits the structure of a nondiscrete dimension-complemented cylindric algebra if and only if it has a direct factor which is denumerable and atomless.

By the elementary theory of Boolean algebras, to say that $\mathfrak{A}$ has a direct factor which is denumerable and atomless is equivalent to saying that $\mathfrak{A}$ is not atomic. Thus, we have proved

Theorem 1. A denumerable Boolean algebra admits the structure of a nondiscrete dimension-complemented cylindric algebra if and only if it is not atomic.

In the discussion which follows we will use an algebraic counterpart of terms in first-order languages. For a full discussion of terms in cylindric algebras the reader is referred to [4]; however, for the present purposes only a few rudimentary notions are needed. An element $x \in A$ will be called "diagonal-like" if it has the following two properties for some $\kappa<\alpha$ :

(1) $c_{\kappa} x=1$, and

(2) $x \cdot s_{\mu}^{\kappa} x \leqslant d_{\kappa \mu}$ for each $\mu \in \alpha-\Delta x$. 
With every diagonal-like element $x \in A$ we associate a term $a$, and (for $x$ satisfying (1) and (2) above), we write $x=d_{\kappa a}$. (In the metalogical interpretation, $d_{\kappa a}$ is the equivalence class of the formula $v_{\kappa}=a$, and (1) and (2) assert the unique existence of $v_{\kappa}$ satisfying $v_{\kappa}=a$. Thus, if $\mathfrak{A}$ is taken to be an algebra of formulas, the "terms" of $\mathfrak{U}$ are all the terms which are explicitly definable in the theory associated with $\mathfrak{A}$.)

The following properties of diagonal-like elements will be relevant to our discussion:

THEOREM 2. If $x$ is any diagonal-like element, then $x \geqslant c_{0}^{\partial} d_{01}$.

Proof. Let $x$ satisfy (1) and (2). By $[1,1.6 .20], c_{\kappa}^{\partial} d_{\kappa \lambda}-x \in Z d \mathfrak{A}$. Thus,

$$
c_{\kappa}^{\partial} d_{\kappa \lambda} \cdot-x=c_{\kappa}^{\partial}\left(c_{\kappa}^{\partial} d_{\kappa \lambda} \cdot-x\right)=c_{\kappa}^{\partial} d_{\kappa \lambda} \cdot-c_{\kappa} x=c_{\kappa}^{\partial} d_{\kappa \lambda} \cdot 0=0 .
$$

Thus, $c_{\kappa}^{\partial} d_{\kappa \lambda} \leqslant x$.

From this theorem, we deduce a useful generalization of $[1$, Theorem 2.3.33]:

Corollary 3. Suppose $\mathfrak{A} \in D c_{\alpha}, \alpha \geqslant 2$, and $\mathfrak{A}$ has a set of generators, $X$, such that all but $n$ elements of $X$ are diagonal-like. Then

(i) $\mid$ At $\mathfrak{A} \mid \leqslant 2^{n}$, and

(ii) $c_{0}^{\partial} d_{01}=\sum A t \mathfrak{A}$.

Proof. By Theorem 2, if $x$ is diagonal-like, then $x \cdot c_{0}^{\partial} d_{01}=c_{0}^{\partial} d_{01}$. The remainder of the argument is exactly as in [1, Theorems 2.3.31 and 2.3.33].

The converse of Theorem 2, which follows next, states that if $x \geqslant c_{0}^{\partial} d_{01}$, then $x$ is generated from the diagonal-like elements of $A$.

THEOREM 4. If $x \geqslant c_{0}^{\partial} d_{01}$, then there is a diagonal-like element $y$ such that $x=-c_{\kappa} c_{\lambda} c_{\mu}\left(y \cdot d_{\kappa \mu}-d_{\kappa \lambda}\right)$.

Proof. Take distinct $\kappa, \lambda, \mu \in \alpha-\Delta x$. Let

$$
y=d_{\kappa \lambda} \cdot d_{\kappa \mu}+d_{\kappa \mu}-d_{\kappa \lambda}-x+d_{\lambda \mu}-d_{\kappa \lambda} \cdot x .
$$

One verifies directly (we omit the simple details) that $c_{\mu} y=1$, and for any $\nu \in \alpha-\Delta y, y \cdot s_{\nu}^{\mu} y \leqslant d_{\mu \nu}$. Thus, $y$ is a diagonal-like element. We note that

$$
c_{\kappa} c_{\lambda} c_{\mu}\left(-d_{\kappa \lambda} \cdot y \cdot d_{\kappa \mu}\right)=c_{\kappa} c_{\lambda}\left[-d_{\kappa \lambda} \cdot c_{\mu}\left(y \cdot d_{\kappa \mu}\right)\right]=c_{\kappa} c_{\lambda}\left(-d_{\kappa \lambda} \cdot s_{\kappa}^{\mu} y\right) .
$$

Now, $s_{\kappa}^{\mu} y=d_{\kappa \lambda}+-d_{\kappa \lambda} \cdot-x$, hence

$$
\begin{aligned}
c_{\kappa} c_{\lambda}\left(-d_{\kappa \lambda} \cdot s_{\kappa}^{\mu} y\right) & =c_{\kappa} c_{\lambda}\left(-d_{\kappa \lambda} \cdot\left[d_{\kappa \lambda}+-d_{\kappa \lambda} \cdot-x\right]\right) \\
& =c_{\kappa} c_{\lambda}\left(-d_{\kappa \lambda} \cdot-x\right)=\left(c_{\kappa} c_{\lambda}-d_{\kappa \lambda}\right) \cdot-x .
\end{aligned}
$$

But by assumption, $-x \leqslant-c_{0}^{\partial} d_{01}=c_{\kappa} c_{\lambda}-d_{\kappa \lambda}$, so $c_{\kappa} c_{\lambda}\left(-d_{\kappa \lambda} \cdot s_{\kappa}^{\mu} y\right)=-x$.

Corollary 5. $\mathfrak{U}$ is generated by its diagonal-like elements iff $c_{0}^{\partial} d_{01}=0$ or $c_{0}^{\partial} d_{01}$ is an atom.

Proof. If $X$ is a set of generators of $\mathfrak{A}$, then (as in the proof of [1, 2.3.31], 
$R l_{c \beta} d_{01} \mathfrak{A}$ is generated by $\left\{x \cdot c_{0}^{\partial} d_{01}: x \in X\right\}$. Thus, if $X$ contains only diagonal-like elements, then by Theorem $2,\left\{x \cdot c_{0}^{\partial} d_{01}: x \in X\right\}=\left\{c_{0}^{\partial} d_{01}\right\}$, hence $c_{0}^{\partial} d_{01}=0$ or $c_{0}^{\partial} d_{01}$ is an atom. Conversely, suppose that $c_{0}^{\partial} d_{01}=0$ or $c_{0}^{\partial} d_{01}$ is an atom. In the first case, $x \geqslant c_{0}^{\partial} d_{01}$ for every $x \in A$; in the second case, either $x \geqslant c_{0}^{\partial} d_{01}$ or $-x \geqslant c_{0}^{\partial} d_{01}$ for every $x \in A$. Thus, by Theorem $4, \mathfrak{A}$ is generated by its diagonal-like elements.

Finally, the following result is of some interest:

THEOREM 6. $\mathfrak{A}$ has a set of generators, $X$, which contains only diagonal-like elements and zero-dimensional elements.

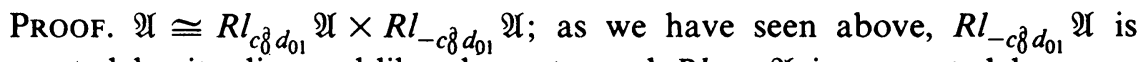
generated by its diagonal-like elements, and $R l_{c \beta} d_{01} \mathfrak{A}$ is generated by zerodimensional elements.

3. Applications to Lindenbaum algebras. The connections between algebraic logic and logic are studied in [2] and [3]. For example, it is proved in [3] that two arbitrary theories are definitionally equivalent iff their associated cylindric algebras are isomorphic. Furthermore, from the discussion in [3], it is clear that if a language has no relation symbols, then its associated cylindric algebra is generated by its diagonal-like elements; and conversely, if the cylindric algebra associated with a theory $T$ is generated by its diagonal-like elements, then $T$ is definitionally equivalent to a theory in a language with no relation symbols. In the sequel, these facts will be used without further explicit mention.

Throughout this section, we will take $\mathscr{A}$ to be the Lindenbaum algebra of formulas, $\mathscr{F}_{T}$, of a first-order theory $T$.

We will show, first, that Corollary 5 yields a necessary and sufficient condition for the eliminability of predicates in favor of functions. We begin by noting the following:

For $\mathfrak{A}=\mathscr{F}_{T}, c_{0}^{\partial} d_{01}=0$ iff $T \vdash \neg\left(\forall v_{0}\right) \quad\left(v_{0}=v_{1}\right)$ iff $T$ has no oneelement models. On the other hand, $c_{0}^{\partial} d_{01}$ is an atom if and only if $R l_{c \beta} d_{01} \mathfrak{A}$ is a two-element Boolean algebra. Now, the discrete cylindric algebra $R l_{c \& d_{01}} \mathfrak{A}$ is the algebra of formulas of the theory of one-element models of $T$, and a theory is complete iff its Boolean algebra of sentences has two elements; thus, $c_{0}^{\partial} d_{01}$ is an atom iff all the one-element models of $T$ are elementarily equivalent. Combining this with Corollary 5, we get

(E) An arbitrary theory $T$ admits elimination of predicates iff $T$ either has no one-element models, or all of its one-element models are elementarily equivalent.

From Theorem 6 we deduce that for any theory $T$, predicate symbols are eliminable in favor of function symbols and propositional constants:

(F) Any theory $T$ is definitionally equivalent to a theory $T^{\prime}$ whose language has no predicate symbols but may have function symbols and propositional constants.

(Note that the propositional constants serve only to axiomatize the class of one-element models of $T$.)

We will now use Corollary 3 to describe the structure of the Lindenbaum algebras of formulas of certain theories. If $T$ has no one-element models, then, as we have already noted, in $\mathscr{F}_{T}, c_{0}^{\partial} d_{01}=0$. Thus, in view of $(\mathrm{A})$, we have 
(G) If $T$ has no one-element models, then $\mathscr{F}_{T}$ is an atomless Boolean algebra.

If $T$ has one-element models, we may use Corollary 3 to deduce

(H) If $T$ admits elimination of all but $n$ predicates, then $\mathcal{F}_{T}$ has $\leqslant 2^{n}$ atoms.

If $T$ is any theory which admits elimination of all but finitely many predicates we can, in fact, find the exact structure of $\mathscr{F}_{T}$. We assume the language $L$ of $T$ is denumerable.

Let $\left\langle P_{i}\right\rangle_{i<n}$ be the sequence of predicate symbols of $L$, and let $\delta_{i}$ be the rank of $P_{i}$ for each $i<n$. It follows from [1, Theorem 2.4.37] that $\mathscr{F}_{T} \cong \mathfrak{B} \times \mathfrak{E}$, where $\mathfrak{B}=R l_{c \beta d_{01}} \mathfrak{A}$ and $\mathfrak{E}=R l_{-c \beta d_{01}} \mathfrak{A}$. We have already seen that $\mathfrak{E}$ belongs to the isomorphism class of denumerable, atomless Boolean algebras, so it remains only to determine the structure of $\mathfrak{B}$. Now, $\mathfrak{B}$ is the algebra of formulas of the theory $T_{1}$ whose nonlogical axioms are those of $T$ together with the formula $\left(\forall v_{0}\right)\left(v_{0}=v_{1}\right)$. It is immediately verified that

$$
T_{1} \vdash P_{i}\left(v_{1}, \ldots, v_{\delta_{i}}\right) \leftrightarrow P_{i}\left(t_{1}, \ldots, t_{\delta_{i}}\right)
$$

for every $i<n$ and all terms $t_{j}$, and $T_{1} \vdash\left(\forall v_{k}\right) F \leftrightarrow F$ for every formula $F$. Thus $\mathfrak{B}$, the algebra of formulas of $T_{1}$, is the same as the algebra of formulas of the theory in the propositional calculus whose propositional variables are $P_{1}, \ldots, P_{n-1}$, and whose axioms are obtained from those of $T_{1}$ by deleting all variables, terms and quantifiers (together with the associated commas and brackets).

\section{BIBLIOGRAPHY}

1. L. Henkin, J. D. Monk and A. Tarski, Cylindric algebras. Part 1. With an introductory chapter: General theory of algebras, Studies in Logic and the Foundations of Math., vol. 64, NorthHolland, Amsterdam, 1971. MR 47 \#3171.

2. L. Henkin and A. Tarski, Cylindric algebras, Proc. Sympos. Pure Math., vol. 2, Amer. Math. Soc., Providence, R.I., 1961, pp. 83-113. MR 23 \# A1564.

3. H.-J. Hoehnke, Zur Strukturgleichheit axiomatischer Klassen, Z. Math. Logik Grundlagen Math. 12 (1966), 69-83. MR 32 \#5499.

4. C. Pinter, Terms in cylindric algebras, Proc. Amer. Math. Soc. 40 (1973), 568-572. MR 48 \#3738.

Department of Mathematics, Bucknell University, Lewisburg, Pennsylvania 17837 